\title{
Recent kaon decay results from NA62
}

\author{
N. Estrada-Tristan*† \\ Universidad Autonoma de San Luis Potosi, Mexico ${ }^{\ddagger}$ \\ E-mail: nora.patricia.estrada.tristan@cern.ch
}

In this paper we describe the NA62 experiment, which is a fixed target experiment at CERN SPS who aims at measuring the Branching Ratio of $K^{+} \rightarrow \pi^{+} v \bar{v}$ but also to study other rare kaon decays. Important results such as advances in measuring the Branching Ratio of $K^{+} \rightarrow \pi^{+} v \bar{v}$, the search for heavy neutral leptons and the search for lepton number violating kaon decays will be shown.

7th Annual Conference on Large Hadron Collider Physics - LHCP2019

20-25 May, 2019

Puebla, Mexico

*Speaker.

${ }^{\dagger}$ for the NA62 Collaboration: R. Aliberti, F. Ambrosino, R. Ammendola, B. Angelucci, A. Antonelli, G. Anzivino, R. Arcidiacono, M. Barbanera, A. Biagioni, L. Bician, C. Biino, A. Bizzeti, T. Blazek, B. Bloch-Devaux, V. Bonaiuto, M. Boretto, M. Bragadireanu, D. Britton, F. Brizioli, M.B. Brunetti, D. Bryman, F. Bucci. T. Capussela, A. Ceccucci, P. Cenci, V. Cerny, C. Cerri, B. Checcucci, A. Conovaloff, P. Cooper, E. Cortina Gil, M. Corvino, F. Costantini, A. Cotta Ramusino, D. Coward, G. D’Agostini, J. Dainton, P. Dalpiaz, H. Danielsson, N. De Simone, D. Di Filippo, L. Di Lella, N. Doble, B. Dobrich, F. Duval, V. Duk, J. Engelfried, T. Enik, N. Estrada-Tristan, V. Falaleev, R. Fantechi, V. Fascianelli, L. Federici, S. Fedotov, A. Filippi, M. Fiorini, J. Fry, J. Fu, A. Fucci, L. Fulton, E. Gamberini, L. Gatignon, G. Georgiev, S. Ghinescu, A. Gianoli, M. Giorgi, S. Giudici, F. Gonnella, E. Goudzovski, C. Graham, R. Guida, E. Gushchin, F. Hahn, H. Heath, T. Husek, O. Hutanu, D. Hutchcroft, L. Iacobuzio, E. Iacopini, E. Imbergamo, B. Jenninger, K. Kampf, V. Kekelidze, S. Kholodenko, G. Khoriauli, A. Khotyantsev, A. Kleimenova, A. Korotkova, M. Koval, V. Kozhuharov, Z. Kucerova, Y. Kudenko, J. Kunze, V. Kurochka, V.Kurshetsov, G. Lanfranchi, G. Lamanna, G. Latino, P. Laycock, C. Lazzeroni, M. Lenti, G. Lehmann Miotto, E. Leonardi, P. Lichard, L. Litov, R. Lollini, D. Lomidze, A. Lonardo, P. Lubrano, M. Lupi, N. Lurkin, D. Madigozhin, I. Mannelli, G. Mannocchi, A. Mapelli, F. Marchetto, R. Marchevski, S. Martellotti, P. Massarotti, K. Massri, E. Maurice, M. Medvedeva, A. Mefodev, E. Menichetti, E. Migliore, E. Minucci, M. Mirra, M. Misheva, N. Molokanova, M. Moulson, S. Movchan, M. Napolitano, I. Neri, F. Newson, A. Norton, M. Noy, T. Numao, V. Obraztsov, A. Ostankov, S. Padolski, R. Page, V. Palladino, C. Parkinson, E. Pedreschi, M. Pepe, M. Perrin-Terrin, L. Peruzzo, P. Petrov, F. Petrucci, R. Piandani, M. Piccini, J. Pinzino, I. Polenkevich, L. Pontisso, Yu. Potrebenikov, D. Protopopescu, M. Raggi, A. Romano, P. Rubin, G. Ruggiero, V. Ryjov, A. Salamon, C. Santoni, G. Saracino, F. Sargeni, V. Semenov, A. Sergi, A. Shaikhiev, S. Shkarovskiy, D. Soldi, V. Sougonyaev, M. Sozzi, T. Spadaro, F. Spinella, A. Sturgess, J. Swallow, S. Trilov, P. Valente, B. Velghe, S. Venditti, P. Vicini, R. Volpe, M. Vormstein, H. Wahl, R. Wanke, B. Wrona, O. Yushchenko, M. Zamkovsky, A. Zinchenko.

$\ddagger$ Also at Universidad de Guanajuato, Departamento de Ciencias e Ingenieria 


\section{Introduction}

Rare kaon decays have proven to be powerful tools for understanding the hadronic internal processes and for testing various models predicting from symmetry violations to new particles.

The NA62 experiment was designed to directly measure the BR of the ultra-rare $K^{+} \rightarrow \pi^{+} v \bar{v}$ decay, but given the high Kaon flux and the different trigger mask operating in the experiment, NA62 is capable of perform plenty of other searches. Recent results from the NA62 experiment will be presented in the following Proceeding.

\section{The NA62 detector}

NA62 is the latest generation kaon experiment at the CERN SPS, aiming to measure the BR of the ultra-rare $K^{+} \rightarrow \pi^{+} v \bar{v}$ decay with $10 \%$ accuracy, which requires a high kinematic rejection power, effective photon and muon rejection and excellent particle identification. The layout of the NA62 detector is shown in Fig. 1. A primary beam of protons extracted from the CERN SPS is used to produce a secondary positive hadron beam with a central momentum of $75 \mathrm{GeV} / \mathrm{c}$ and $1 \%$ momentum spread (rms). Kaons from the secondary beam are tagged by a differential Cherenkov counter. Beam particle momenta are measured by a silicon pixel detector (GTK). A 75 $\mathrm{m}$ long fiducial decay volume in vacuum (FV) follows the last GTK station. In the decay region, a spectrometer made of 4 chambers with straw tubes in vacuum and a dipole magnet measures track directions and momenta (STRAW). Pions and muons are separated by a ring imaging Cherenkov (RICH) detector and muon detectors (MUV1-3). A system of calorimeters (LKr, LAVs, IRC, SAC) detects photons at different acceptances. The nominal instantaneous beam particle rate is $750 \mathrm{MHz}$, mostly due to $\pi^{+}(70 \%)$, protons $(23 \%)$ and $K^{+}(6 \%)$. About $11 \%$ of the kaons decay in the FV, leading to about $5 \mathrm{MHz}$ nominal $K^{+}$rate in the FV. Details of the NA62 beam and detector can be found in [1].

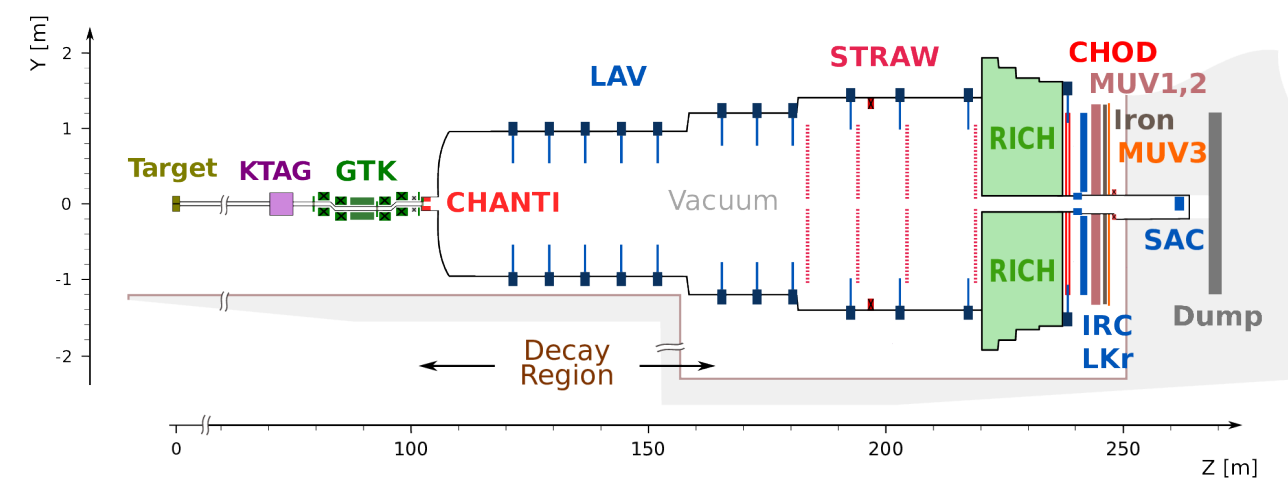

Figure 1: NA62 schematic side view of beam line and detector.

\section{Kaon rare decay $K^{+} \rightarrow \pi^{+} v \bar{v}$}

The $K^{+} \rightarrow \pi^{+} v \bar{v}$ decay is a Flavor Changing Neutral Current process forbidden at tree level in the Standard Model (SM) and proceeding through penguin and box diagrams. It is highly sup- 
pressed due to the quadratic GIM mechanism and the small value of the CKM element $\left|V_{t d}\right|$, which makes it an ideal channel to search for physics beyond the SM (BSM) [2-7]. The SM prediction is $\mathscr{B}\left(K^{+} \rightarrow \pi^{+} v \bar{v}\right)=(8.4 \pm 1.0) \times 10^{-11}$ [8] where the dominant sources of uncertainties are the CKM parameters.

\subsection{Signal selection}

The topology of the signal consists of only an incoming $K^{+}$, and an outgoing $\pi^{+}$. The detected incoming track positively identified as a kaon and the outgoing (downstream) tracks associated in time are used to reconstruct the decay vertex at their closest distance of approach. The longitudinal position of the vertex is required to be located within a $50 \mathrm{~m}$ long decay region. The identification of the downstream particle is performed by combining the RICH measurement with the output of a multivariate classifier using the $\mathrm{LKr}$ and the hadronic calorimeters, providing a $\pi^{+}$identification efficiency of $64 \%$ for a $\mu^{+}$suppression of $1 \times 10^{8}$. The remaining background consists mostly of the $K^{+} \rightarrow \pi^{+} \pi^{0}$ decay, which is suppressed by a factor of $3 \times 10^{8}$ thanks to the hermetic photon veto system (LAV, LKr, IRC, SAC) which rejects with an efficiency better than the $99.99 \%$ level the photons inside the detector.

The main kinematic variable for this analysis is the missing mass squared $m_{m i s s}^{2}=\left(P_{K}-P_{\pi}\right)^{2}$, which is computed in three ways using different combination of $P_{K}$ and $P_{\pi}$ measurements, to protect against mis-reconstruction of the momenta. The kaon momentum $\overrightarrow{p_{K}}$ can be measured directly with the GTK. The pion momentum $\vec{p}_{\pi}$ can be measured either with the STRAW or with the RICH. The analysis is performed in two separate regions in the $m_{m i s s}^{2}$ variables, on either side of the $K^{+} \rightarrow \pi^{+} \pi^{0}$ peak (R1 as $[0-0.01] \mathrm{GeV}^{2} / \mathrm{c}^{4}$ and $\mathrm{R} 2$ as $[0.026-0.068] \mathrm{GeV}^{2} / \mathrm{c}^{4}$ ). These limits are defined by the missing mass squared resolution which is $10^{-3} \mathrm{GeV}^{2} / \mathrm{c}^{4}$. In both regions, the $\pi^{+}$momentum must be in the range $15-35 \mathrm{GeV} / \mathrm{c}$ to ensure at least $40 \mathrm{GeV}$ of electromagnetic energy in the calorimeters and to optimize the RICH $\pi^{+} / \mu^{+}$separation.

\subsection{Results from the 2016 data}

The signal acceptance A is determined from Monte-Carlo (MC) simulations, giving $\mathrm{A}=1 \%$ in $\mathrm{R} 1$ and $\mathrm{A}=3 \%$ in $\mathrm{R} 2$. The number of kaon decays in the FV is measured from a sample of $K^{+} \rightarrow$ $\pi^{+} \pi^{0}$ decays selected with control triggers and is approximated to be $N_{K(2016)}=(1.21 \pm 0.04) \times$ $10^{11}$. The single event sensitivity is $S E S=1 /\left(A \cdot \varepsilon \cdot N_{K}\right)=\left(3.15 \pm 0.01_{\text {stat }} \pm 0.24_{\text {syst }}\right)$, where $\varepsilon$ takes into account the inefficiencies due to the trigger and the random veto caused by accidental activity. Multiplying this number by the SM branching fraction of the $K^{+} \rightarrow \pi^{+} v \bar{v}$ decay, the expected number of signal events is $0.267 \pm 0.001_{\text {stat }} \pm 0.020_{\text {sys }} \pm 0.032_{\text {ext }}$. The contribution of the $K^{+} \rightarrow \pi^{+} \pi^{-} e^{+} v$ decay to the background is evaluated using MC data with a sample of 600 decays, which show a good agreement across the validation samples. The other background sources are estimated using data-driven techniques and validated on control regions around the signal regions. The upstream background is due to accidental activity in the detector and interactions of the beam particles in the upstream detectors. It is effectively suppressed by geometrical cuts and by the cuts in the kaon and pion tracks vertex. The background contributions are summarized in Table 1.

In March 2017 we declared the analysis finished and opened the blinded box. One event was observed in R2, as shown in Fig. 2, corresponding to a p-value of $15 \%$ for the background only hypothesis and an observed upper limit of $\mathscr{B}\left(K^{+} \rightarrow \pi^{+} v \bar{v}\right)<14 \times 10^{-10}$ at $95 \%$ CL [9]. 


\begin{tabular}{l|l}
\hline Process & Expected events in signal regions \\
\hline$K^{+} \rightarrow \pi^{+} \pi^{0}(\gamma)$ & $0.064 \pm 0.007_{\text {stat }} \pm 0.006_{\text {syst }}$ \\
$K^{+} \rightarrow \mu^{+} v(\gamma)$ & $0.20 \pm 0.003_{\text {stat }} \pm 0.006_{\text {syst }}$ \\
$K^{+} \rightarrow \pi^{+} \pi^{-} e^{+} v$ & $0.0013_{-0.012}^{+0.017} \pm 0.009_{\text {stat }}$ \\
$K^{+} \rightarrow \pi^{+} \pi^{+} \pi^{0}$ & $0.002 \pm 0.001_{\text {stat }} \pm 0.002_{\text {syst }}$ \\
$K^{+} \rightarrow \pi^{+} \gamma \gamma$ & $<0.001$ \\
Upstream Background & $\left.0.05_{-0.030}^{+0.090}\right|_{\text {stat }}$ \\
Total background & $\left.0.152_{-0.033}^{+0.092}\right|_{\text {stat }} \pm 0.013_{\text {syst }}$ \\
\hline
\end{tabular}

Table 1: Summary of the background estimates summed over the two signal regions for the analysis of the 2016 data-set [9].

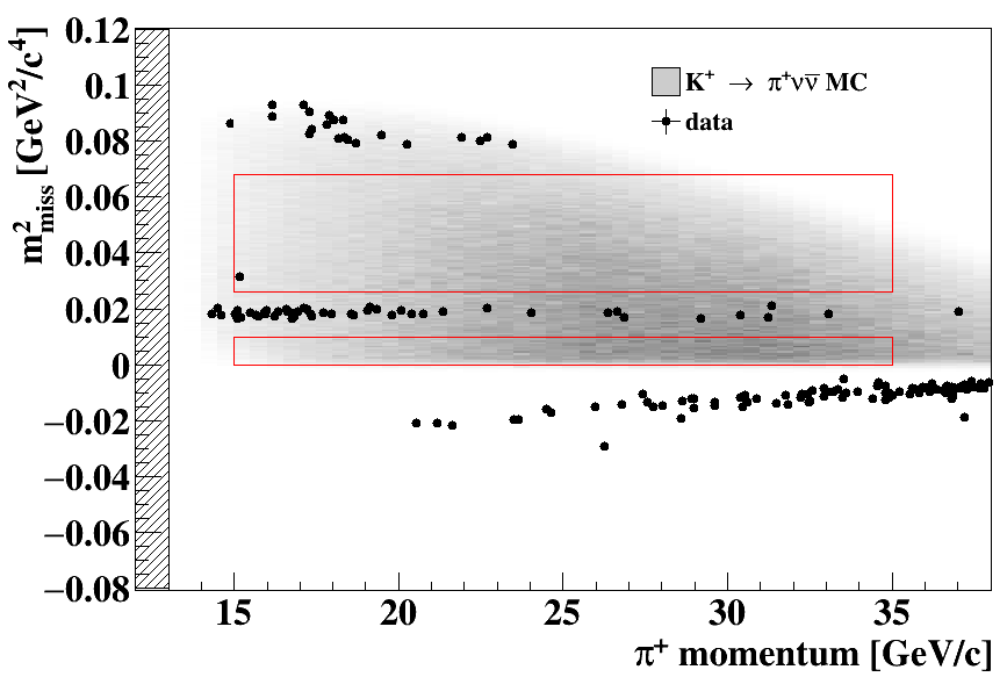

Figure 2: Reconstructed $m_{\text {miss }}^{2}$ of the 2016 data as a function of the $\pi^{+}$momentum for events satisfying the $K^{+} \rightarrow \pi^{+} v \bar{v}$ selection [9]. The gray area corresponds to the distribution of MC signal events. The red contours define the signal regions.

\subsection{Preliminary Results 2017 Data}

The analysis with 2017 data is ongoing, the signal and control areas are covered and will be unblinded soon. Being largely similar to the 2016 analysis except that we expect a factor 10 of improvement from statistics. Signal-over-background does not degrade with intensity. Considering $N_{K(2017)}=(1.3 \pm 0.1) \times 10^{12}$ and $S E S=(0.34 \pm 0.04) \times 10^{-10}$, the expected number of SM events in 2017 is $2.5 \pm 0.4$ (Fig. 3).

\section{Dark photons}

An extension of the SM adding a new $U(1)$ gauge-symmetry sector is one of the proposed solutions to explain the abundance of dark matter in the universe. In the simplest realization of this scenario [10,11], the vector mediator field $A^{\prime}$ (dark photon) of mass $M_{A^{\prime}}$ associated to the new $U(1)$ interacts with the SM through a kinetic mixing term whose strength is given by the parameter $\varepsilon$. 


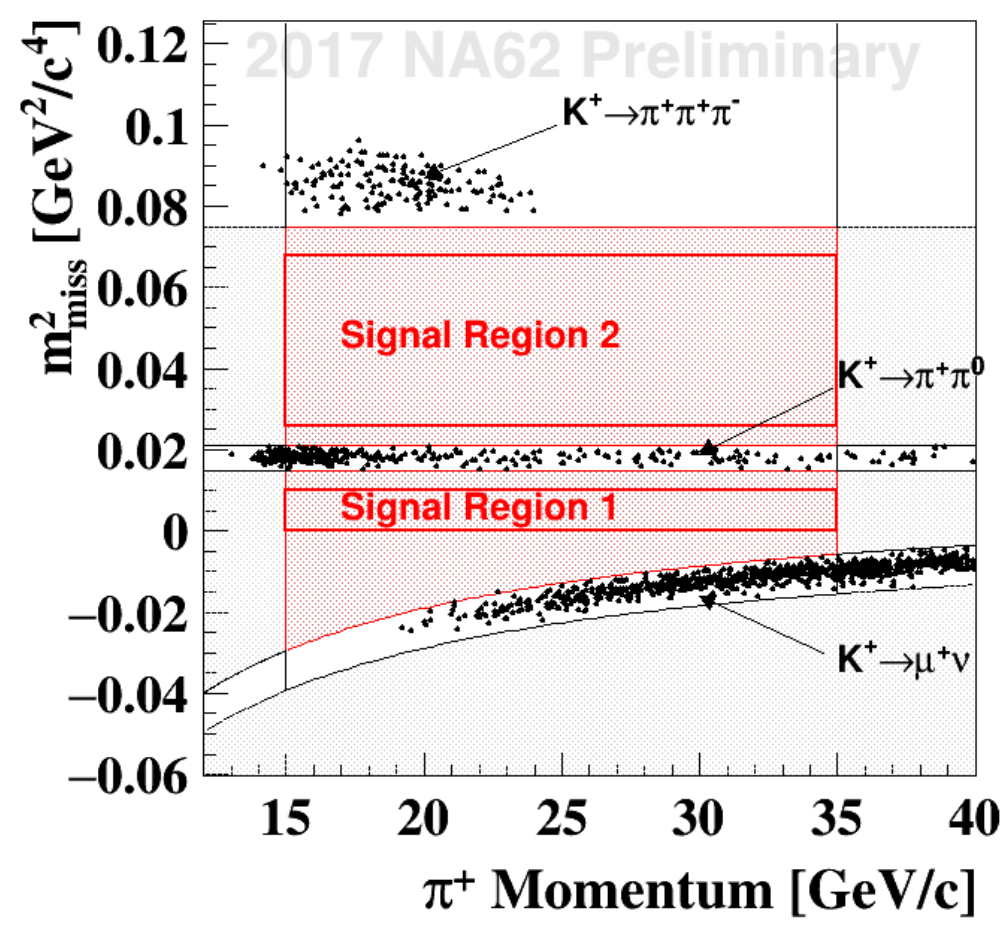

Figure 3: Reconstructed $m_{m i s s}^{2}$ of the 2017 data as a function of the $\pi^{+}$momentum for events satisfying the $K^{+} \rightarrow \pi^{+} v \bar{v}$ selection. The background analysis is under study and the signal regions are still blinded.

In such a case, it is possible for the $A^{\prime}$ to be produced in the decay chain $K^{+} \rightarrow \pi^{+} \pi^{0}, \pi^{0} \rightarrow A^{\prime} \gamma$, presumming that the $A^{\prime}$ does not decay into SM particles inside the detector, a missing energy signature might be a hint of its presence.

A search for an invisible dark photon $A^{\prime}$ has been performed. The NA62 photon-veto rejection, precisely studied in the context of the analyses for $K^{+} \rightarrow \pi^{+} v \bar{v}$ and for the search for $\pi^{0}$ decays to invisible final states, is paramount for the $A^{\prime}$ search (Fig. 6).

Detailed information about this analysis was reported [12] at this conference.

\section{Heavy Neutral Leptons (HNL)}

The Standard Model (SM) of particle physics requires an extension to explain, among other things, neutral lepton masses. One of this SM extensions, the Neutrino Minimal Standard Model $(v$ MSM), proposes three massive, right-handed "sterile" neutrinos, also called heavy neutral leptons (HNL), which mix with the ordinary light "active" neutrinos, explaining also dark matter and baryon asymmetry in the universe (BAU) [23]. The lightest HNL, with mass of $\mathscr{O}\left(10 \mathrm{keV} / c^{2}\right)$ ), is a dark matter candidate. The other two HNL have masses of $\mathscr{O}\left(1 \mathrm{GeV} / c^{2}\right)$, produce the SM neutrino masses through the see-saw mechanisms and introduce extra $\mathrm{CP}$ violating phases to account for BAU. A mixing matrix U describes the interactions between HNL and SM leptons. The mass range and the small mixing angles predicted in the $v$ MSM make HNL long lived, with mean free paths of $\mathscr{O}(10 \mathrm{~km})$ or longer, and production branching fractions of $\mathscr{O}\left(10^{-10}\right)$ or smaller. 


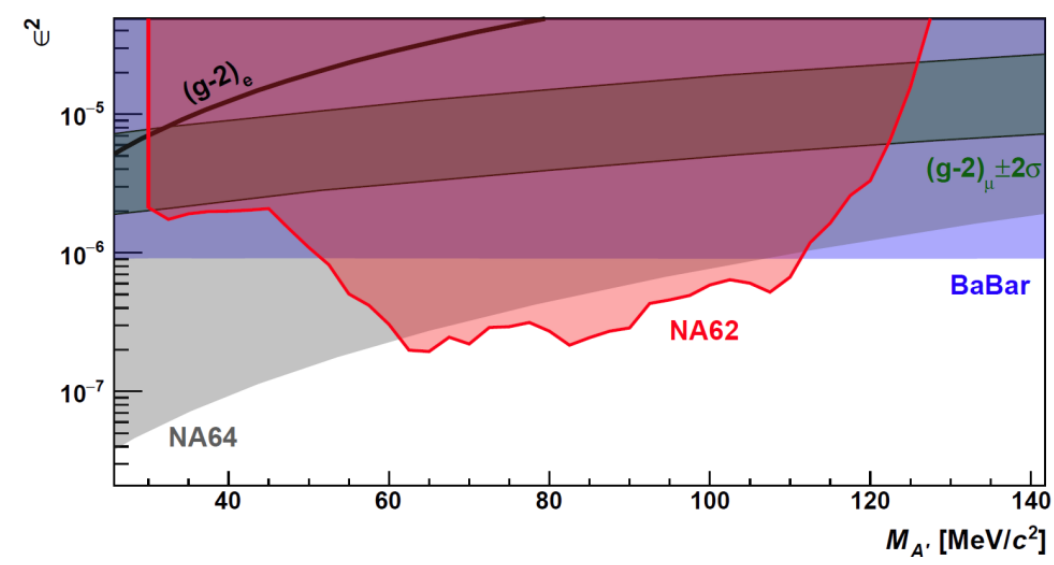

Figure 4: Upper limit at 90\% CL from NA62 [13] (red region) in the $\varepsilon^{2}$ vs $M_{A^{\prime}}$ plane with $A^{\prime}$ decaying into invisible final states. The limits from the BaBar [14] (blue) and NA64 [15] (light gray) experiments are shown. The green band shows the region of the parameter space corresponding to an explanation of the discrepancy between the measured [16] and expected values of the anomalous muon magnetic moment $(g-2)$ [17] in terms of a contribution from the $A^{\prime}$ in the quantum loops [18, 19]. The region above the black line is excluded by the agreement of the anomalous magnetic moment of the electron $(g-2)_{e}$ with its expected value [20-22].

The mixing between HNL (denoted $N$ below) and active light neutrinos gives rise to HNL production in meson decays, including $K^{+} \rightarrow \ell^{+} N(\ell=e, \mu)$ [24,25].

NA62 perform a search for $K^{+} \rightarrow \ell^{+} N$ decays in the HNL mass range $170-448 \mathrm{MeV} / c^{2}$ using a data sample collected with a minimum bias trigger at $1 \%$ of the nominal beam intensity, during the first physics data-taking in 2015. The obtained upper limits on $\left|U_{\ell 4}\right|^{2}$ complement, and improve on those obtained in earlier HNL production searches [26-30].

Upper limits have been established at the level between $10^{-6}$ and $10^{-7}$ on the HNL mixing parameters $\left|U_{e 4}\right|^{2}$ and $\left|U_{\mu 4}\right|^{2}$ in the ranges $170-448 \mathrm{MeV} / \mathrm{c}^{2}$ and $250-373 \mathrm{MeV} / \mathrm{c}^{2}$, respectively [31] Fig. 5. This improves on the previous limits from HNL production searches over the whole mass range considered for $\left|U_{e 4}\right|^{2}$, and above $m_{N}=300 \mathrm{MeV} / c^{2}$ for $\left|U_{\mu 4}\right|^{2}$ [26].

See also the results reported in this congress talk in this congress [12].

\section{Searches For Lepton Number Violating $K^{+}$Decays (LFV)}

The total Lepton Number is a conserved quantity in the SM. However, Lepton Number Violation (LNV) is predicted in some BSM models. The kaon decays $K^{+} \rightarrow \pi^{-} \ell^{+} \ell^{+}[\ell=e, \mu]$ would then be allowed and proceed via an intermediate mediator such as a Majorana Neutrino $[32,33]$. Previous experimental results set the following limits at $90 \% \mathrm{CL}$ on the branching fraction of such channels: $\mathscr{B}\left(K^{+} \rightarrow \pi^{-} e^{+} e^{+}\right)<6.4 \times 10^{-10}$ [34] and $\mathscr{B}\left(K^{+} \rightarrow \pi^{-} \mu^{+} \mu^{+}\right)<8.6 \times 10^{-11}$ [35].

NA62 perform a search for these processes using a part of the $K^{+}$decay in flight data sample collected in 2016-18, corresponding to about $30 \%$ of the total statistics accumulated during that period. A blind analysis procedure was adopted and an event selection, similar for both channels, 


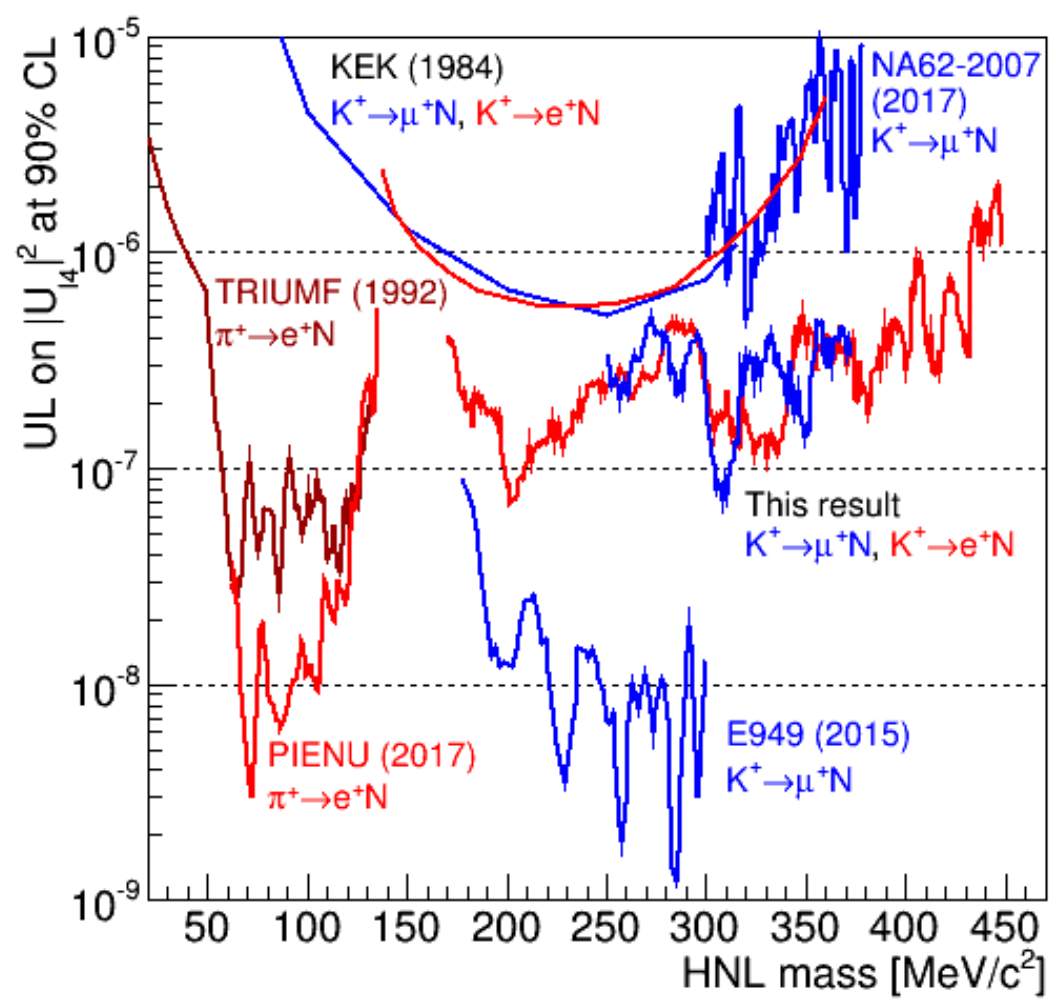

Figure 5: Upper limits at 90\% CL on $\left|U_{4}\right|^{2}$ [31] obtained for each assumed HNL mass compared to the limits established by earlier HNL production searches in $\pi^{+}$decays: TRIUMF (1992) [26], PIENU (2017) [27] and $K^{+}$decays: KEK (1984) [28], E949 (2015) [29], NA62-2007 (2017) [30].

was developed. In both cases the corresponding $K^{+} \rightarrow \pi^{+} \ell^{+} \ell^{-}$SM channel was used for normalization, which results in the first order cancellation of systematic uncertainties. In the $\ell=e$ case, the number of kaon decays $N_{K}=(2.14 \pm 0.07) \times 10^{11}$, the signal acceptance of $4.98 \%$ and the expected number of background events of $0.16 \pm 0.03$ for 0 observed signal events result in an upper limit on the branching fraction $\mathscr{B}\left(K^{+} \rightarrow \pi^{-} e^{+} e^{+}\right)<2.2 \times 10^{-10}$ at $90 \% \mathrm{CL}$ (Fig. 6 left). In the $\ell=\mu$ case, the number of kaon decays $N_{K}=(7.94 \pm 0.23) \times 10^{11}$, the signal acceptance of $9.81 \%$ and the expected number of background events of $0.91 \pm 0.41$ for 1 observed signal event result in an upper limit on the branching fraction $\mathscr{B}\left(K^{+} \rightarrow \pi^{-} \mu^{+} \mu^{+}\right)<4.2 \times 10^{-11}$ at $90 \% \mathrm{CL}$ (Fig. 6 right) [36].

\section{Conclusions}

In this paper we presented in a condensed way the latest results in kaon research perform, by the NA62 Collaboration. All the results presented in this proceeding are already published. NA62 continue collecting data during 2018 run. At the moment, this data is being prepared for analysis, which will give a more precise measurement of the $\mathscr{B}\left(K^{+} \rightarrow \pi^{+} v \bar{v}\right)$. Other analysis are being performed as well, covering all the allowed and not allowed $K^{+}$decays, as well as searches in the dark sector and using the experiment in Beam Dump Mode. 

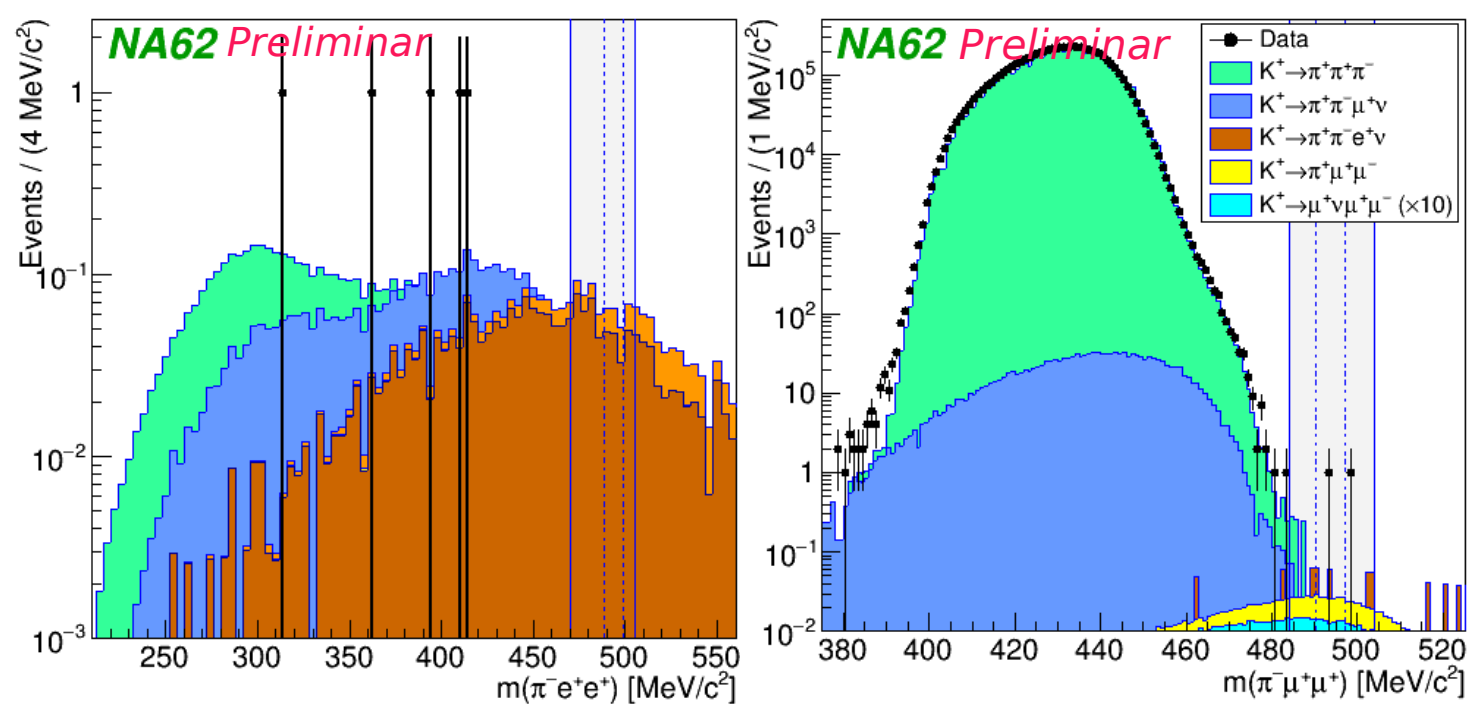

Figure 6: Reconstructed mass spectra for LNV. Data are overlayed with background estimates based on simulations. The shaded vertical bands indicate the region masked during the analysis, including the LNV signal region bounded by dashed lines [36].

\section{References}

[1] NA62 collaboration, The Beam and detector of the NA62 experiment at CERN, JINST 12 (2017) P05025 [1703.08501].

[2] M. Blanke, A. J. Buras, B. Duling, K. Gemmler and S. Gori, Rare K and B Decays in a Warped Extra Dimension with Custodial Protection, JHEP 03 (2009) 108 [0812 . 3803].

[3] G. Isidori, F. Mescia, P. Paradisi, C. Smith and S. Trine, Exploring the flavour structure of the MSSM with rare K decays, JHEP 08 (2006) 064 [hep-ph/ 0604074 ].

[4] T. Blažek and P. Maták, Left-left squark mixing, $K^{+} \rightarrow \pi^{+} v \bar{v}$ and minimal supersymmetry with large tan $\beta$, Int. J. Mod. Phys. A29 (2014) 1450162 [1410.0055].

[5] A. J. Buras, D. Buttazzo and R. Knegjens, $K \rightarrow \pi v \bar{v}$ and $\varepsilon^{\prime} / \varepsilon$ in simplified new physics models, JHEP 11 (2015) 166 [1507.08672].

[6] M. Blanke, A. J. Buras and S. Recksiegel, Quark flavour observables in the Littlest Higgs model with T-parity after LHC Run 1, Eur. Phys. J. C76 (2016) 182 [1507.06316].

[7] M. Bordone, D. Buttazzo, G. Isidori and J. Monnard, Probing Lepton Flavour Universality with $K \rightarrow \pi v \bar{v}$ decays, Eur. Phys. J. C77 (2017) 618 [1705.10729].

[8] A. J. Buras, D. Buttazzo, J. Girrbach-Noe and R. Knegjens, $K^{+} \rightarrow \pi^{+} v \bar{v}$ and $K_{L} \rightarrow \pi^{0} v \bar{v}$ in the Standard Model: status and perspectives, JHEP 11 (2015) 033 [1503.02693].

[9] NA62 collaboration, First search for $K^{+} \rightarrow \pi^{+} v \bar{v}$ using the decay-in-flight technique, Phys. Lett. B791 (2019) 156 [1811.08508].

[10] B. Holdom, Two U(1)'s and Epsilon Charge Shifts, Phys. Lett. 166B (1986) 196.

[11] B. Batell, M. Pospelov and A. Ritz, Exploring Portals to a Hidden Sector Through Fixed Targets, Phys. Rev. D80 (2009) 095024 [0 906 . 5614 ]. 
[12] J. Engelfried, Dark sector searches in NA62 andother non-LHC experiments, in LHCP19, these proceeding, 2019.

[13] NA62 collaboration, Search for production of an invisible dark photon in $\pi^{0}$ decays, JHEP 05 (2019) $182[1903.08767]$.

[14] BABAR collaboration, Search for Invisible Decays of a Dark Photon Produced in $e^{+} e^{-}$Collisions at BaBar, Phys. Rev. Lett. 119 (2017) 131804 [1702.03327].

[15] NA64 collaboration, Search for vector mediator of Dark Matter production in invisible decay mode, Phys. Rev. D97 (2018) 072002 [1710.00971].

[16] Muon G-2 collaboration, Final Report of the Muon E821 Anomalous Magnetic Moment Measurement at BNL, Phys. Rev. D73 (2006) 072003 [hep-ex/ 0602035$].$

[17] Particle Data Group collaboration, Review of Particle Physics, Phys. Rev. D98 (2018) 030001.

[18] P. Fayet, U-boson production in e+e- annihilations, psi and Upsilon decays, and Light Dark Matter, Phys. Rev. D75 (2007) 115017 [hep-ph/ 0702176$].$

[19] M. Pospelov, Secluded U(1) below the weak scale, Phys. Rev. D80 (2009) 095002 [0811 . 1030].

[20] D. Hanneke, S. Fogwell and G. Gabrielse, New Measurement of the Electron Magnetic Moment and the Fine Structure Constant, Phys. Rev. Lett. 100 (2008) 120801 [0801.1134].

[21] R. Bouchendira, P. Clade, S. Guellati-Khelifa, F. Nez and F. Biraben, New determination of the fine structure constant and test of the quantum electrodynamics, Phys. Rev. Lett. 106 (2011) 080801 [1012.3627].

[22] T. Aoyama, M. Hayakawa, T. Kinoshita and M. Nio, Tenth-Order QED Contribution to the Electron g-2 and an Improved Value of the Fine Structure Constant, Phys. Rev. Lett. 109 (2012) 111807 [1205.5368].

[23] T. Asaka and M. Shaposhnikov, The nuMSM, dark matter and baryon asymmetry of the universe, Phys. Lett. B620 (2005) 17 [hep-ph/ 0505013$].$

[24] R. E. Shrock, New Tests For, and Bounds On, Neutrino Masses and Lepton Mixing, Phys. Lett. 96B (1980) 159.

[25] R. E. Shrock, General Theory of Weak Leptonic and Semileptonic Decays. 1. Leptonic Pseudoscalar Meson Decays, with Associated Tests For, and Bounds on, Neutrino Masses and Lepton Mixing, Phys. Rev. D24 (1981) 1232.

[26] D. I. Britton et al., Improved search for massive neutrinos in pi+ $\rightarrow$ e+ neutrino decay, Phys. Rev. D46 (1992) R885.

[27] PIENU collaboration, Improved search for heavy neutrinos in the decay $\pi \rightarrow e v$, Phys. Rev. D97 (2018) 072012 [1712.03275].

[28] T. Yamazaki et al., Search for Heavy Neutrinos in Kaon Decay, .

[29] E949 collaboration, Search for heavy neutrinos in $K^{+} \rightarrow \mu^{+} v_{H}$ decays, Phys. Rev. D91 (2015) 052001 [1411.3963].

[30] NA62 collaboration, Search for heavy neutrinos in $K^{+} \rightarrow \mu^{+} v_{\mu}$ decays, Phys. Lett. B772 (2017) 712 [1705.07510].

[31] NA62 collaboration, Search for heavy neutral lepton production in $K^{+}$decays, Phys. Lett. B778 (2018) 137 [1712.00297]. 
[32] L. S. Littenberg and R. Shrock, Implications of improved upper bounds on $\mid$ Delta L $\mid=2$ processes, Phys. Lett. B491 (2000) 285 [hep-ph/ 0005285 ].

[33] A. Atre, T. Han, S. Pascoli and B. Zhang, The Search for Heavy Majorana Neutrinos, JHEP 05 (2009) 030 [0901.3589].

[34] R. Appel et al., Search for lepton flavor violation in K+ decays, Phys. Rev. Lett. 85 (2000) 2877 [hep-ex/0006003].

[35] NA48/2 collaboration, Searches for lepton number violation and resonances in $K^{ \pm} \rightarrow \pi \mu \mu$ decays, Phys. Lett. B769 (2017) 67 [1612. 04723 ].

[36] NA62 collaboration, Searches for lepton number violating $K^{+}$decays, Phys. Lett. B797 (2019) $134794[1905.07770]$. 